\title{
Low-Cost Brain-Computer Interface Using the Emotiv Epoc Headset Based on Rotating Vanes
}

\author{
Mesut Melek $^{1^{*}}$, Negin Manshouri² ${ }^{2}$ Temel Kayikcioglu ${ }^{2}$ \\ ${ }^{1}$ Department of Electronics and Automation, Gumushane University, Gumushane 29100, Turkey \\ ${ }^{2}$ Department of Electrical and Electronics Engineering, Faculty of Engineering, Karadeniz Technical University, Trabzon \\ 61080, Turkey
}

Corresponding Author Email: mesutmelek@gumushane.edu.tr

https://doi.org/10.18280/ts.370516

Received: 7 June 2020

Accepted: 13 October 2020

\section{Keywords:}

EEG, Emotiv Epoc headset, brain-computer interface, rotating vanes, information transfer rate

\begin{abstract}
In the brain-computer interface system (BCI), electroencephalography (EEG) signals are converted into digital signals and analyzed, allowing direct communication between humans and the electronic devices around them. The convenience of the user and the speed of communication with the surrounding devices are the most important challenges of BCI systems. The Emotiv Epoc headset minimizes the discomfort of the user thanks to its wet electrodes and easy handling. In the continuation of our previous works, in this paper, we developed our BCI system based on the gaze at the rotating vanes using the inexpensive Emotiv Epoc headset. In addition to user comfort, our design has an acceptable mean accuracy rate (ACC) and mean information transfer rate (ITR) compared to similar systems.
\end{abstract}

\section{INTRODUCTION}

Throughout Brain-computer interface (BCI) systems allow people with physical disabilities to contact people around them and control external electronic devices such as wheelchairs and prostheses. The purpose of these systems is translating brain signals into commands that the person intends, without the need for any muscle of the disabled person to work [1]. For many researchers, these systems are the only possible solution for physically disabled people [2, 3].

Electroencephalography (EEG) is an electrical signal with a high temporal resolution and a very small amplitude (in the $\mu \mathrm{V}$ range) produced by neuronal activations in the brain [4]. EEG recording is more easily done than recording other brain signals such as Functional magnetic resonance imaging (FMRI) [5], and its low risk and low cost have contributed to its preference in BCI systems [6]. Emotiv Epoc is one of the cheapest and increasingly used EEG recorders in terms of usability [7, 8]. It is an EEG device that is prepared rapidly and is inexpensive and user-friendly, with light and wet electrodes. This device was not introduced for research purposes but for gaming and entertainment, with a number of software packages that can detect the user's emotions and facial expressions and control the objects in a virtual world. However, it has become increasingly popular due to its flexibility and the wide range of suites it offers [9]. The Emotiv Epoc device has 14 electrodes and two reference electrodes placed in the 10-10 international EEG electrode placement system. The internal sampling rate of the device is $2048 \mathrm{~Hz}$, but the data are then sampled down to $128 \mathrm{~Hz}$ before accessing the system. With Emotiv Epoc, it takes less than 5 minutes to obtain a clear EEG signal, and it feels comfortable for the user. This is very good compared to the preparation time of other gel devices. For these reasons, Emotiv Epoc is user-friendly and suitable for practical applications.
Although the Emotiv Epoc headset has restrictions in terms of signal quality, it is gaining popularity in BCI research. It has been used in various BCI applications because of its accessibility and portability for consumers and researchers [10]. Some studies have compared Emotiv Epoc with other devices $[11,12]$. It is clear that the low-cost Emotiv Epoc yields satisfactory results since the accuracy rates of the systems do not drop significantly when using this equipment. As a result, Emotiv's performance has been proven competent for BCI systems [9], and due to the reasons mentioned, Emotiv is a tool that promises to bring a simple, affordable BCI into people's lives [11].

In the literature, there are many BCI systems with the Emotiv Epoc device. Tong et al. [13] in a study based on the P300 paradigm achieved an $88.75 \%$ accuracy rate and 7.17 bits/min information transfer rate (ITR). This system was tested on 20 people in two locations, on the subway and in the office. Results on the subway were poorer than in Office. A steady-state visually evoked potential (SSVEP)-based BCI system was recommended by Soroush et al. [14]. The goal of their study was to design a BCI system that did not require any training phase and could, therefore, be used immediately by new users. The accuracy rate of this system with the Emotiv Epoc was $94.85 \%$, and ITR was 1.5 bits per trial. The study by Mijani et al. [15] tested an offline speller system on three people. The accuracy rate and ITR of this P300-based study were calculated for single, dual, and triple rapid serial visual presentation, 78\%, 63\%, 64\%, and 3.65, 7.72, and 11.5 bits/min, respectively. In a study conducted in 2019 [16], the combination of different colors used for SSVEP was tested, and a $\sim 90 \%$ accuracy rate and $\sim 8$ bits/min ITR were calculated. Moreover, in a study based on the motor imagery paradigm [17], the accuracy rate was calculated as $93.6 \%$. This study was conducted on seven people in the offline mode and used deep learning methods for classifications. The ITR of the 
system has not been computed.

The aim of this study was to develop a user-friendly, completely independent BCI system by using only the brain signals of the disabled, thereby increasing the users' quality of life by enabling them to interact with the surrounding environment. Each of the common paradigms used in BCI systems has its own disadvantages. In the P300 and SSVEP paradigms, the user has to look at the lights constantly blinking. This is an irritating issue for long-term users and may even cause eye diseases. In the motor imagery paradigm, the user has to consider the movement of his/her limbs constantly. We proposed a BCI system based on rotating vanes in our previous studies $[18,19]$ to solve these problems. However, the EEG device used in our studies was wired and with gel. In the present study, we tested our system on the Emotiv Epoc device, which has a lower quality signal and is cheap. The BCI system based on rotating vanes with the Emotiv Epoc device will provide more comfort for users.

The organization of this article is as follows: Materials and methods are explained after the Introduction section. This section involves data description, pre-processing, feature extraction, classification, and performance metrics in BCI systems, in that order. In the third section, the results are described, and in the fourth and fifth sections, discussion and conclusion are given, respectively.

\section{MATERIALS AND METHODS}

\subsection{Data description}

As mentioned earlier, in order to overcome the problems of popular BCI paradigms, we proposed a BCI system based on rotating vanes in our previous studies. Thanks to this system, the user can avoid looking at flashing lights or thinking of constantly moving limbs. We used the interface designed through Matlab 2014a in our previous works. This interface is given in Figure 1. As it appears, four "A" letters were written in white on a black screen, and a red vane was placed on top of each. The speed and direction of rotation of the vanes are as follows:

- Vane 1 (top left) rotates in a counterclockwise manner every five seconds.

- Vane 2 (top right) rotates in a counterclockwise manner every one second.

- Vane 3 (bottom left) rotates in a clockwise manner every five seconds.

- Vane 4 (bottom right) rotates in a clockwise manner every one second.

In this way, the options presented according to the user's needs will be located under each vane and it will be sufficient for the user to look at the vane on the desired option.

EEG signals were taken by the Emotiv Epoc EEG device from five participants (four men and one woman) aged 27 to 32 years at the Electrical and Electronics Engineering Department, Karadeniz Technical University. The experiments were performed with the registration number 24237859-640 according to the rules of the Faculty of Medicine Ethics Committee, Karadeniz Technical University (Turkey). The participants had no previous experience of using any BCI systems and could withdraw at any time during the experiment without any pressure on them. The Emotiv Epoc device sampled the EEG signal through 14 electrodes at 128 samples/sec. The participants were seated at a distance of $1 \mathrm{~m}$ in front of a 32-inch monitor, and after a few seconds of rest, recordings commenced. The participants were asked to look at each vane for 125 seconds. This process was started and finished with a beep sound. After hearing the second beep sound, and before starting watching the next vane, the participants were given a resting time of about 1 minute. In this way, the data set was recorded from a total of five participants. Then, the data set was divided into 6-sec, 4-sec, and 2-sec epochs, with four seconds, two seconds, and one second of overlapping, respectively (Table 1). For example, the EEG signal in a single channel, which is 125 seconds for each vane, was separated into 6-second segments by four seconds of overlapping, resulting in a total of 59 epochs.

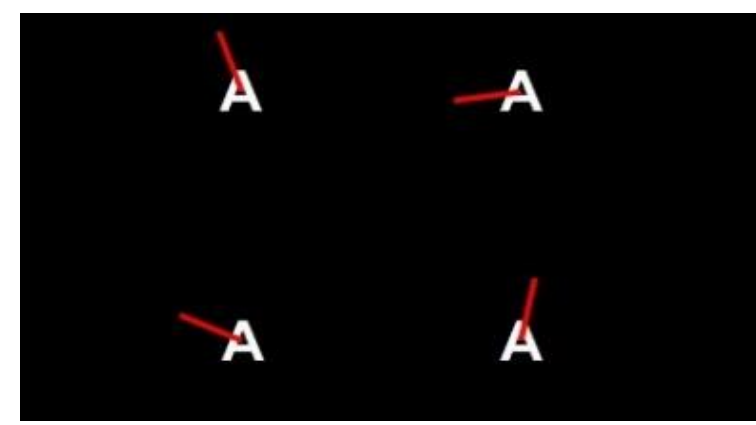

Figure 1. Rotating vanes designed by Matlab 2014a [19]

Table 1. Description of the data set for one participant in an EEG channel

\begin{tabular}{ccc}
\hline $\begin{array}{c}\text { Epoch } \\
\text { duration }\end{array}$ & $\begin{array}{c}\text { Overlapping } \\
\text { (sec.) }\end{array}$ & $\begin{array}{c}\text { Epoch number for each } \\
\text { vane }\end{array}$ \\
\hline $6-\mathrm{sec}$ & 4 & 59 \\
$4-\mathrm{sec}$ & 2 & 60 \\
$2-\mathrm{sec}$ & 1 & 120 \\
\hline
\end{tabular}

\subsection{Pre-processing}

The amplitude of the signals can directly affect the classification performance. Therefore, the min-max normalization method was applied in each epoch, as shown in Eq. (1), to reduce the effect of amplitude.

$$
X_{N}=\frac{x-\min (x)}{\max (x)-\min (x)}
$$

Here, $x$ and $X_{N}$ are the original epoch and normalized epoch indicator, respectively. Furthermore, processing of EEG signals requires special approaches such as filtering [20]. After normalization, each epoch was passed through a $0.1-45 \mathrm{~Hz}$ bandpass Butterworth filter. The order of the filter was determined as three by trial and error, and Matlab filtfilt command was used to reduce the phase shift to zero.

\subsection{Feature extraction}

The power spectrum density (PSD) analysis provides basic information on how power is distributed as a function of frequency. In this study, the Welch method was adopted to calculate PSD [21]. A short summary of the Welch method can be described as follows: In the first step, each epoch is divided into parts of a certain length with certain overlaps. In the second step, by applying a window to each part, the relevant periodogram is calculated, i.e., a Fast Fourier Transform (FFT) 
is applied to the windowed data. Finally, in the third step, the spectral density is estimated by averaging over the modified periodograms. The spectrum estimate obtained is scaled to calculate the PSD. In our study, a smooth Hamming window [22] w(n) is applied to each part of data based on Eq. (2).

$$
\mathrm{W}(\mathrm{n})=0.54-0.46 \cos (2 \mathrm{n} \pi / \mathrm{L})
$$

where, $\mathrm{n}=0,1,2 \ldots \mathrm{L}-1$, and $\mathrm{L}$ denotes the length of each part. After calculating the PSD of each epoch, features were extracted by considering the five frequency rhythms of the EEG signal [23]. For this, the areas formed between the PSD graph and the $\mathrm{x}$-axis for delta $(0-4 \mathrm{~Hz})$, theta $(4-7 \mathrm{~Hz})$, alpha $(8-12 \mathrm{~Hz})$, beta $(13-30 \mathrm{~Hz})$, and gamma $(30-45 \mathrm{~Hz})$ bands was calculated by Matlab trapz command, and thus 5 features were obtained from each epoch. A feature vector of an epoch was created by bringing these features obtained from 14 channels side by side.

\subsection{Classification}

An algorithm trained with labelled training samples to identify unknown samples is called a classifier. The support vector machine (SVM) [24], linear discriminant analysis (LDA) [25], k-nearest neighbors (k-NN) [26], and Ada boost [26] are classifiers used on Emotiv-based BCI systems. SVM (linear, quadratic, cubic, medium Gaussian), linear discriminant, and ensemble subspace discriminant were employed in this study.

\subsection{Performance metrics in BCI systems}

\subsubsection{Accuracy rate (ACC)}

The accuracy rate is the most used metric in a classification process. This metric is obtained by dividing the number of correctly identifying samples of the system by the number of all correctly and incorrectly defined samples by using the Eq. (3) [27]. Calculation of ACC is usually performed according to two popular strategies: K-fold cross-validation (K-FCV) strategy and holdout strategy.

$$
A C C=\frac{\text { Number of samples classified correctly }}{\text { Total samples }}
$$

K-fold cross-validation (K-FCV) strategy: In this strategy, the data set is divided into $\mathrm{K}$ equal set; the $\mathrm{K}-1$ subdivision is used as the training data set; and a subdivision is utilized as the testing data set [28]. All subdivisions are used as a testing data set once, and the accuracy rate is calculated each time. The accuracy rate of the system is the average of the accuracy rates obtained [29, 30]. In the K-FCV strategy, $\mathrm{K}$ is chosen as many as the number of samples to reduce the risk of unlucky splitting. In this strategy, called leave one-out cross-validation (LOO-CV), one sample is employed as a testing set, and the remaining samples are used as a training set. Here, we used the LOO-CV strategy for the computation of ACC.

Holdout strategy: The holdout strategy is frequently used to evaluate the performance of BCI systems. In this strategy, the data set is divided into two sets of training and testing. The classifier is trained with the training set and the model is created. Then, the model is tested with the test dataset. In general, researchers create different training and testing sets by mixing the data set several times to demonstrate the stability of their systems, and in this way, they obtain the overall accuracy rate of the system by calculating the average of the calculated accuracy rates each time.

\subsubsection{ITR}

The value of ITR is very influential, as BCI systems interact directly with patients [31]. The ITR is the most comprehensive performance metric of BCI systems. This metric derives from the principles of information theory and summarizes the three important parameters of the BCI system with a single value. These parameters are the accuracy rate of the system, the time it takes for a selection to be recognized in the system, and the number of tasks in each selection. According to Shannon's ITR, ITR is defined by Eq. (4) [32].

$$
B_{t}=\log _{2} K+p \log _{2} p+(1-p) \log _{2}\left(\frac{1-p}{K-1}\right)
$$

where, $K$ indicates how many rights the user has in each selection, and $p$ represents the system's ACC. The unit of $B_{t}$ is bits per trial and in minutes can be expressed by Eq. (5).

$$
I T R=60 * \frac{B t}{T}
$$

where, $T$ is the time it takes for a selection to be recognized in the system. In our study, we have four vanes so $\mathrm{K}$ is 4 . Also, $T$ is 6,4 , and 2 when we used 6-sec, 4-sec, and 2-sec epochs, respectively. For more details and proofs of ITR based on information theory, could be referred to the studies [33, 34].

\section{RESULTS}

As previously mentioned, after EEG signals were recorded, they were divided into, 6-sec, 4-sec, and 2-sec epochs. After normalization and filtering of epochs, PSD was calculated by the Welch method. It is known that EEG signals have five frequency rhythms: delta band $(0-4 \mathrm{~Hz})$, theta band $(4-7 \mathrm{~Hz})$, alpha band $(8-12 \mathrm{~Hz})$, beta-band $(13-30 \mathrm{~Hz})$, and gamma band $(30-49 \mathrm{~Hz})$. In the PSD graph, the area of the areas that make up these bands with the x-axis was calculated as the features of each epoch, and thus 5 features were obtained from each epoch. These features obtained from 14 channels were brought side by side to form the feature vector of an epoch. These feature vectors were classified into six classifiers with two strategies. The accuracy rates and ITRs of 6-sec epochs obtained based on the LOO-CV strategy are shown in Table 2. Also, in Tables 3 and Table 4, the results of 4-sec and 2-sec epochs are given according to this strategy. The participants were named S1, S2, S3, S4, and S5, and in the last column of the tables, for five participants, the average of each metric was calculated.

In the holdout strategy, $75 \%$ of the data set was used for the training of the classifier and $25 \%$ for the testing of the created model. To reduce the risk of unlucky splitting, this procedure was repeated 10 times and the average accuracy rate was calculated. Results for 6-sec, 4-sec, and 2-sec epochs were given in Tables 5, 6, and 7, respectively. In addition, the Cohen's kappa coefficient [35] of the system and the sensitivity of each class were presented in Figures A1, A2, and A3 for 6-sec, 4-sec, and 2-sec epochs in Appendix, respectively. 
Table 2. Accuracy rates and ITRs for 6-sec epochs based on LOO-CV strategy; the highest value in each metric is indicated in boldface

\begin{tabular}{cccccccc}
\hline Classifier & Metric & S1 & S2 & S3 & S4 & S5 & AVG. \\
\hline Linear discriminant & ACC & 0.9322 & 0.8686 & 0.9873 & 0.9322 & 0.8390 & 91.18 \\
& ITR & 15.35 & 12.31 & 18.82 & 15.35 & 11.08 & 14.58 \\
Linear SVM & ACC & 0.8644 & 0.8814 & 0.9831 & 0.9280 & 0.8475 & 90.08 \\
& ITR & 12.13 & 12.87 & 18.49 & 15.12 & 11.42 & 14.01 \\
Quadratic SVM & ACC & 0.9280 & 0.8941 & 0.9915 & 0.9661 & 0.8814 & 93.22 \\
& ITR & 15.12 & 13.45 & 19.16 & 17.33 & 12.87 & 15.58 \\
Cubic SVM & ACC & 0.9364 & 0.8814 & 0.9873 & 0.9576 & 0.9237 & $\mathbf{9 3 . 7 2}$ \\
& ITR & 15.58 & 12.87 & 18.82 & 16.80 & 14.90 & $\mathbf{1 5 . 7 9}$ \\
Medium Gaussian SVM & ACC & 0.8559 & 0.8051 & 0.9958 & 0.9407 & 0.8432 & 88.81 \\
& ITR & 11.77 & 9.79 & 19.54 & 15.81 & 11.25 & 13.63 \\
Ensemble Subspace discriminant & ACC & 0.9153 & 0.8983 & 0.9915 & 0.9619 & 0.8983 & 93.3 \\
& ITR & 14.47 & 13.64 & 19.16 & 17.06 & 13.64 & 15.60 \\
\hline
\end{tabular}

Table 3. Accuracy rates and ITRs for 4-sec epochs based on LOO-CV strategy; the highest value in each metric is indicated in boldface

\begin{tabular}{cccccccc}
\hline Classifier & Metric & S1 & S2 & S3 & S4 & S5 & AVG. \\
\hline Linear discriminant & ACC & 0.7875 & 0.7917 & 0.9167 & 0.8542 & 0.7542 & 0.8208 \\
& ITR & 13.75 & 13.97 & 21.81 & 17.54 & 12.09 & 15.83 \\
Linear SVM & ACC & 0.8000 & 0.7875 & 0.9292 & 0.8542 & 0.7708 & 0.8283 \\
& ITR & 14.42 & 13.75 & 22.78 & 17.54 & 12.90 & 16.27 \\
Quadratic SVM & ACC & 0.8292 & 0.7958 & 0.9417 & 0.8625 & 0.8125 & 0.8483 \\
& ITR & 16.04 & 14.19 & 23.80 & 18.07 & 15.10 & 17.44 \\
Cubic SVM & ACC & 0.8500 & 0.8083 & 0.9458 & 0.8583 & 0.8167 & $\mathbf{0 . 8 5 5 8}$ \\
& ITR & 17.29 & 14.87 & 24.15 & 17.80 & 15.33 & $\mathbf{1 7 . 8 8}$ \\
Medium Gaussian SVM & ACC & 0.7667 & 0.6958 & 0.9167 & 0.8417 & 0.7875 & 0.8016 \\
& ITR & 12.70 & 9.47 & 21.81 & 16.78 & 13.75 & 14.9 \\
Ensemble Subspace discriminant & ACC & 0.8208 & 0.8167 & 0.9500 & 0.8667 & 0.7917 & 0.8491 \\
& ITR & 15.57 & 15.33 & 24.52 & 18.33 & 13.97 & 17.54 \\
\hline
\end{tabular}

Table 4. Accuracy rates and ITRs for 2-sec epochs based on LOO-CV strategy; the highest value in each metric is indicated in boldface

\begin{tabular}{cccccccc}
\hline Classifier & Metric & S1 & S2 & S3 & S4 & S5 & AVG. \\
\hline Linear discriminant & ACC & 0.7583 & 0.7521 & 0.8063 & 0.7625 & 0.7438 & 0.7645 \\
& ITR & 24.57 & 23.97 & 29.51 & 24.98 & 23.18 & 25.24 \\
Linear SVM & ACC & 0.7792 & 0.7458 & 0.8271 & 0.7729 & 0.7396 & 0.7729 \\
& ITR & 26.65 & 23.38 & 31.85 & 26.02 & 22.80 & 26.13 \\
Quadratic SVM & ACC & 0.7812 & 0.7500 & 0.8354 & 0.7708 & 0.8104 & 0.7895 \\
& ITR & 26.86 & 23.77 & 32.82 & 25.81 & 29.97 & 27.84 \\
Cubic SVM & ACC & 0.7417 & 0.7667 & 0.8187 & 0.7563 & 0.8208 & $\mathbf{0 . 7 8 0 8}$ \\
& ITR & 22.99 & 25.39 & 30.90 & 24.37 & 31.13 & $\mathbf{2 6 . 9 5}$ \\
Medium Gaussian SVM & ACC & 0.7396 & 0.7146 & 0.8417 & 0.7458 & 0.7833 & 0.765 \\
Ensemble Subspace discriminant & ITR & 22.80 & 20.55 & 33.56 & 23.38 & 27.08 & 25.47 \\
& ACC & 0.7708 & 0.7646 & 0.8187 & 0.7812 & 0.7542 & 0.7779 \\
& ITR & 25.81 & 25.19 & 30.90 & 26.86 & 24.17 & 26.58 \\
\hline
\end{tabular}

Table 5. Accuracy rates and ITRs for 6-sec epochs based on holdout strategy; the highest value in each metric is indicated in boldface

\begin{tabular}{cccccccc}
\hline Classifier & Metric & S1 & S2 & S3 & S4 & S5 & AVG. \\
\hline Linear discriminant & ACC & 0.8839 & 0.8179 & 0.9768 & 0.8964 & 0.8214 & 0.8792 \\
& ITR & 13.12 & 10.38 & 17.42 & 13.63 & 10.60 & 13.03 \\
Linear SVM & ACC & 0.8214 & 0.8518 & 0.9750 & 0.8893 & 0.8357 & 0.8746 \\
& ITR & 10.48 & 11.65 & 17.58 & 13.35 & 11.09 & 12.82 \\
Quadratic SVM & ACC & 0.8821 & 0.8732 & 0.9839 & 0.9464 & 0.8714 & $\mathbf{0 . 9 1 1 4}$ \\
& ITR & 13.00 & 12.56 & 18.08 & 16.21 & 12.69 & $\mathbf{1 4 . 5 0}$ \\
Cubic SVM & ACC & 0.8875 & 0.8464 & 0.9786 & 0.9357 & 0.8768 & 0.9050 \\
& ITR & 13.34 & 11.55 & 17.82 & 15.62 & 12.84 & 14.23 \\
Medium Gaussian SVM & ACC & 0.7964 & 0.7929 & 0.9786 & 0.8982 & 0.8054 & 0.8542 \\
& ITR & 9.61 & 9.41 & 17.65 & 13.74 & 9.96 & 12.07 \\
Ensemble Subspace & ACC & 0.8865 & 0.8821 & 0.9721 & 0.9304 & 0.8839 & 0.9110 \\
discriminant & ITR & 13.22 & 13.06 & 17.29 & 15.35 & 13.24 & 14.42 \\
\hline
\end{tabular}


Table 6. Accuracy rates and ITRs for 4-sec epochs based on holdout strategy; the highest value in each metric is indicated in boldface

\begin{tabular}{cccccccc}
\hline Classifier & Metric & S1 & S2 & S3 & S4 & S5 & AVG. \\
\hline Linear discriminant & ACC & 0.7150 & 0.7750 & 0.9033 & 0.7917 & 0.7117 & 0.7793 \\
& ITR & 10.52 & 13.21 & 20.94 & 14.07 & 10.33 & 13.81 \\
Linear SVM & ACC & 0.7667 & 0.7883 & 0.8950 & 0.8450 & 0.7617 & 0.8113 \\
& ITR & 12.75 & 13.85 & 20.57 & 17.09 & 12.60 & 15.37 \\
Quadratic SVM & ACC & 0.7767 & 0.7950 & 0.9083 & 0.8600 & 0.8083 & 0.8296 \\
& ITR & 13.36 & 14.24 & 20.66 & 18.05 & 15.01 & 16.26 \\
Cubic SVM & ACC & 0.7800 & 0.7800 & 0.9100 & 0.8533 & 0.8133 & 0.8273 \\
& ITR & 13.49 & 13.42 & 20.78 & 17.59 & 15.34 & 16.12 \\
Medium Gaussian SVM & ACC & 0.7183 & 0.6900 & 0.9083 & 0.8267 & 0.7667 & 0.7820 \\
& ITR & 10.62 & 9.40 & 21.43 & 15.96 & 12.80 & 14.04 \\
Ensemble Subspace discriminant & ACC & 0.7817 & 0.8167 & 0.9300 & 0.8617 & 0.8000 & $\mathbf{0 . 8 3 8 0}$ \\
& ITR & 13.56 & 15.41 & 23.03 & 18.14 & 14.48 & $\mathbf{1 6 . 9 2}$ \\
\hline
\end{tabular}

Table 7. Accuracy rates and ITRs for 2 -sec epochs based on holdout strategy; the highest value in each metric is indicated in boldface

\begin{tabular}{cccccccc}
\hline Classifier & Metric & S1 & S2 & S3 & S4 & S5 & AVG. \\
\hline Linear discriminant & ACC & 0.7133 & 0.7408 & 0.7825 & 0.7417 & 0.7567 & 0.7470 \\
& ITR & 20.61 & 23.19 & 27.12 & 23.10 & 24.49 & 23.70 \\
Linear SVM & ACC & 0.7250 & 0.7500 & 0.7992 & 0.7475 & 0.7317 & 0.7506 \\
& ITR & 21.57 & 23.88 & 28.89 & 23.72 & 22.18 & 24.05 \\
Quadratic SVM & ACC & 0.7800 & 0.7433 & 0.8042 & 0.7358 & 0.7842 & $\mathbf{0 . 7 6 9 5}$ \\
& ITR & 26.94 & 23.31 & 29.40 & 22.63 & 27.23 & $\mathbf{2 5 . 9 0}$ \\
Cubic SVM & ACC & 0.7825 & 0.7583 & 0.8000 & 0.7150 & 0.7808 & 0.7673 \\
& ITR & 27.09 & 24.66 & 28.99 & 20.68 & 26.90 & 25.67 \\
Medium Gaussian SVM & ACC & 0.7517 & 0.7008 & 0.8117 & 0.7175 & 0.7475 & 0.7458 \\
Ensemble Subspace discriminant & ITR & 24.13 & 19.52 & 30.31 & 20.95 & 23.58 & 23.70 \\
& ACC & 0.7367 & 0.7525 & 0.7925 & 0.7467 & 0.7775 & 0.7611 \\
& ITR & 22.70 & 24.16 & 28.31 & 23.62 & 26.60 & 25.08 \\
\hline
\end{tabular}

In all of the sections of this study, the Linear discriminant classifier, the covariance structure was selected as full. In Linear, Quadratic, Cubic SVM classifier Box constraint level was selected 1, and kernel scale mode was Auto. In the Medium Gaussian SVM classifier box constraint level was 1 and the kernel scale was selected as 8.4. In all SVM classifiers, one vs. one method was used for the upgrade of the binary classifier to a multi-class classifier. In Ensemble Subspace discriminant number of learners was 30 and subspace dimensions were selected as 35 . These parameters were selected by the try and error method and are constant in all sections of this study.

\section{DISCUSSIONS}

A BCI system based on the rotating vanes with the Emotiv Epoc device was proposed and evaluated in 6-sec, 4-sec, and 2 -sec epochs according to two strategies. According to the LOO-CV strategy, the cubic SVM classifier gave better results than the other five classifiers. The average accuracy rate obtained with this strategy for the 6-sec epochs was calculated as $93.72 \%$, and the average ITR of the system was 15.79 bits $/ \mathrm{min}$. The average accuracy rates were $85.58 \%$ and $78.08 \%$ in 4-sec and 2-sec epochs, and the ITR of the system was computed as 17.88 and 26.95 bits/min, respectively.

In the results obtained with the hold-out strategy, which is more common in offline studies of BCI systems, quadratic SVM is the most successful classifier in 6-sec and 2-sec epochs. The average accuracy rates obtained for the 6-sec and 2 -sec epochs were $91.14 \%$ and $76.95 \%$, and the speed of the system equaled 14.50 and $25.90 \mathrm{bits} / \mathrm{min}$, respectively. The Ensemble Subspace discriminant classifier was more successful in 4-sec epochs. The average accuracy rate and ITR of the system were calculated as $83.80 \%$ and $16.92 \mathrm{bits} / \mathrm{min}$, respectively. Successful in 6 -sec and 2 -sec epochs, the quadratic SVM followed this classifier with little difference (82.96\% and $16.26 \mathrm{bits} / \mathrm{min})$.

In most Emotiv-based BCI systems (such as [36] and [37]), the system's accuracy rate and ITR were not computed. Still, extensive studies are available in the literature. In a study based on the SSVEP paradigm, Brennan et al. [38] used Emotiv Epoc and reached a 79.2\% accuracy rate and 15.23 bits/min ITR. In a 2012 study [11], the accuracy rate of the system in the offline mode was calculated as $82.99 \% \pm 4.98 \%$. In this SSVEP-based study, the speed of the system was given as $28.06 \pm 6.45 \mathrm{bits} / \mathrm{min}$. Four participants contributed to this study. The accuracy rate of the system in the online mode was $95.83 \% \pm 3.59 \%$ and the ITR was $20.97 \pm 0.37 \mathrm{bits} / \mathrm{min}$. The results of other studies are close to these values [14] or even less $[13,15,16]$. When we compare the paradigm problems and the results of these studies with the proposed system's paradigm and results, it becomes clear that the proposed system provides more comfort for users, and the design has an acceptable ACC and ITR compared to similar systems.

\section{CONCLUSION}

In EEG-based BCI systems, EEG signals are analyzed and the secret thoughts of the user are translated into certain commands; in this way, the user can communicate with his/her surroundings. Although BCI faces many challenges in terms of applicability, usability, and accuracy rate, it is the future of the human-machine interface and the only communication destination for paralyzed people. The convenience of the user 
and the speed of communication with the surrounding devices are the most important challenges of BCI systems. The Emotiv Epoc headset minimizes the discomfort of the user with its wet electrodes and easy usage. To continue our previous works, in this article, we developed our BCI system based on rotating vanes using the Emotiv Epoc headset. The system's offline accuracy rate and speed were calculated as $\sim 77 \%, 84 \%, 92 \%$, and 26, 17, $15 \mathrm{bits} / \mathrm{min}$ for the 2-sec, 4-sec, and 6-sec epochs, respectively. In the next study, we have planned to increase the accuracy rate of 2 -sec epochs by testing different feature extraction and classification methods, and to test the system in a realistic environment by using the proposed BCI system as a control terminal in the online mode.

\section{REFERENCES}

[1] Kaur, B., Singh, D., Roy, P.P. (2018). EEG based emotion classification mechanism in BCI. Procedia Comput. Sci., Elsevier B.V., 132: 752-758. https://doi.org/10.1016/j.procs.2018.05.087

[2] Wolpaw, J.R., Birbaumer, N., Heetderks, W.J., McFarland, D.J., Peckham, P.H., Schalk, G., Donchin, E., Quatrano, L.A., Robinson, C.J., Vaughan, T.M. (2000). Brain-computer interface technology: A review of the first international meeting. IEEE Transactions on Rehabilitation Engineering, 8(2): 164-173. https://doi.org/10.1109/TRE.2000.847807

[3] Kelly, S.P., Lalor, E.C., Finucane, C., McDarby, G., Reilly, R.B. (2005). Visual spatial attention control in an independent brain-computer interface. IEEE Transactions on Biomedical Engineering, 52 (9): 158896. https://doi.org/10.1109/TBME.2005.851510

[4] Rafik, D., Larbi, B. (2019). Autoregressive modeling based empirical mode decomposition (EMD) for epileptic seizures detection using EEG signals. Traitement Du Signal, 36(3): 273-279. https://doi.org/10.18280/ts.360311

[5] Valente, G., Kaas, A.L., Formisano, E., Goebel, R. (2019). Optimizing fMRI experimental design for MVPA-based BCI control: Combining the strengths of block and event-related designs. NeuroImage, 186: 369 381

https://doi.org/10.1016/j.neuroimage.2018.10.080.Epub 2018 Nov 1

[6] Chen, C.H., Ho, M.S., Shyu, K.K., Hsu, K.C., Wang, K.W., Lee, P.L. (2014). A noninvasive brain computer interface using visually-induced near-infrared spectroscopy responses. Neuroscience Letters, 580: 22 26. https://doi.org/10.1016/j.neulet.2014.07.042

[7] Stamps, K., Hamam, Y. (2010). Towards inexpensive BCI control for wheelchair navigation in the enabled environment - A hardware survey. Lect. Notes Comput. Sci. (Including Subser. Lect. Notes Artif. Intell. Lect. Notes Bioinformatics), Springer, Berlin, Heidelberg, pp. 336-345. https://doi.org/10.1007/978-3-642-15314-3_32

[8] Alzu'bi, H.S., Al-Zubi, N.S., Al-Nuaimy, W. (2011). Toward inexpensive and practical brain computer interface. 2011 Developments in E-systems Engineering, Dubai, pp. 98-101. https://doi.org/10.1109/DeSE.2011.116

[9] Maskeliunas, R., Damasevicius, R., Martisius, I., Vasiljevas, M. (2016). Consumer-grade EEG devices: Are they usable for control tasks? PeerJ, 4: e1746. https://doi.org/10.7717/peerj.1746
[10] Masood, N., Farooq, H. (2017). Emotiv-based low-cost brain computer interfaces: A survey. Adv. Intell. Syst. Comput., Springer, Cham, pp. 133-142. https://doi.org/10.1007/978-3-319-41691-5 12

[11] Liu, Y., Jiang, X., Cao, T., Wan, F., Mak, P.Ü., Mak, P.I., Vai, M.I. (2012). Implementation of SSVEP based BCI with Emotiv EPOC. In: Proc. IEEE Int. Conf., Virtual Environ. Human-Computer Interfaces, Meas. Syst., pp. 34-37. https://doi.org/10.1109/VECIMS.2012.6273184

[12] Van Vliet, M., Robben, A., Chumerin, N., Manyakov, N. V., Combaz, A., Van Hulle, M.M. (2012). Designing a brain-computer interface controlled video-game using consumer grade EEG hardware. 2012 ISSNIP Biosignals and Biorobotics Conf, pp. 1-6. https://doi.org/10.1109/BRC.2012.6222186

[13] Tong, J., Peng, Z., Ran, X., Lei, D. (2015). The portable P300 dialing system based on tablet and Emotiv Epoc headset. Annu. Int. Conf. IEEE Eng. Med. Biol. Soc. EMBS, Institute of Electrical and Electronics Engineers Inc. pp. 566-569. https://doi.org/10.1109/EMBC.2015.7318425

[14] Soroush, P.Z., Shamsollahi, M.B. (2019). A non-userbased BCI application for robot control. 2018 IEEEEMBS Conf. Biomed. Eng. Sci., Sarawak, Malaysia, pp. 36-41. https://doi.org/10.1109/IECBES.2018.8626701

[15] Mijani, A.M., Shamsollahi, M.B., Hassani, M.S., Jalilpour, S. (2019). Comparison between single, dual and triple rapid serial visual presentation paradigms for P300 speller. 2018 IEEE Int. Conf. Bioinforma. Biomed, Madrid, Spain, pp. 2635-2638. https://doi.org/10.1109/BIBM.2018.8621505

[16] Li, X.D., Wang, X.J., Wong, C.M., Wen, R.W., Wan, F., $\mathrm{Hu}, \mathrm{Y}$. (2019). Influence of stimuli color combination on online SSVEP-based BCI performance. In: 2019 IEEE Int. Conf. Comput. Intell. Virtual Environ. Meas. Syst. Appl. CIVEMSA 2019 - Proc., Institute of Electrical and Electronics Engineers Inc. https://doi.org/10.1109/CIVEMSA45640.2019.9071623

[17] Zhang, X., Yao, L., Zhang, S., Kanhere, S., Sheng, M., Liu, Y.H. (2019). Internet of Things meets braincomputer interface: A unified deep learning framework for enabling human-thing cognitive interactivity. IEEE Internet of Things Journal, 6(2): 2084-2092. https://doi.org/10.1109/JIOT.2018.2877786

[18] Maleki, M., Manshouri, N., Kayikcioglu, T. (2018). Fast and accurate classifier-based brain-computer interface system using single channel EEG data. In: 26th IEEE Signal Process. Commun. Appl. Conf. SIU 2018, Institute of Electrical and Electronics Engineers Inc., pp. 1-4. https://doi.org/10.1109/SIU.2018.8404376

[19] Maleki, M., Manshouri, N., Kaykcioglu, T. (2017). A new brain-computer interface system based on classification of the gaze on four rotating vanes. International Journal of Computer Science and Information Security (IJCSIS), 15(2): 437-443.

[20] Dimitrov, G.P., Kostadinova, I.S., Panayotova, G., Petrova, P., Aleksiev, K., Oleksii, B., Shevchenko, V. (2019). Using mixed linear programming to classify BCI signals submission. In: 2019 IEEE 60th Annu. Int. Sci. Conf. Power Electr. Eng. Riga Tech. Univ. RTUCON, Riga, Latvia, pp. 1-5. https://doi.org/10.1109/RTUCON48111.2019.8982368

[21] Welch, P.D. (1967). The use of fast fourier transform for the estimation of power spectra: A method based on time 
averaging over short, modified periodograms. IEEE Transactions on Audio and Electroacoustics, 15(2): 7073. https://doi.org/10.1109/TAU.1967.1161901

[22] Xiong, Q., Zhang, X., Wang, W.F., Gu, Y.H. (2020). A parallel algorithm framework for feature extraction of EEG signals on MPI. Computational and Mathematical Methods in Medicine, 2020: 9812019. https://doi.org/10.1155/2020/9812019

[23] Kayikcioglu, T., Maleki, M., Eroglu, K. (2015). Fast and accurate PLS-based classification of EEG sleep using single channel data. Expert Systems with Applications, 42(21):

$7825-7830$ https://doi.org/10.1016/j.eswa.2015.06.010

[24] Wang, J.F. (2015). A Framework for The Integration of the Emotiv EEG system into the NeuCube Spiking Neural Network Environment for Robotics Control. Auckland University of Technology.

[25] Duvinage, M., Castermans, T., Petieau, M., Hoellinger, T., Cheron, G., Dutoit, T. (2013). Performance of the Emotiv Epoc headset for P300-based applications. BioMedical Engineering Online, 12(1): 56. https://doi.org/10.1186/1475-925X-12-56

[26] Pham, T.D., Tran, D. (2012). Emotion recognition using the emotiv EPOC device. In: Lect. Notes Comput. Sci. (Including Subser. Lect. Notes Artif. Intell. Lect. Notes Bioinformatics), Springer, Berlin, Heidelberg, pp. 394399. https://doi.org/10.1007/978-3-642-34500-5_47

[27] Shi, M.H., Liu, X.Q., Zhou, C.L., Chao, F., Liu, C., Jiao, X.Z., An, Y.F., Nwachukwu, S.E., Jiang, M. (2018). Towards portable SSVEP-based brain-computer interface using Emotiv EPOC and mobile phone. In: Proc. - 2018 10th Int. Conf. Adv. Comput. Intell. ICACI 2018, Institute of Electrical and Electronics Engineers Inc., pp. 249-253. https://doi.org/10.1109/ICACI.2018.8377615

[28] Ayado, V.G.B., Dungca, C.J.N., Lazam, S.A., Pereira, M.N.L., Tan, J.R.O., Prado, S.V. (2019). Development of an EEG-based motor imagery brain-computer interface system for lower limb assistive technologies. In: 2018 IEEE 10th Int. Conf. Humanoid, Nanotechnology, Inf. Technol. Commun. Control. Environ. Manag. HNICEM 2018, pp. 1-6. https://doi.org/10.1109/HNICEM.2018.8666350

[29] Nanthini, B.S., Santhi, B. (2017). Electroencephalogram signal classification for automated epileptic seizure detection using genetic algorithm. Journal of Natural Science, Biology and Medicine, 8(2): 159-166. https://doi.org/10.4103/jnsbm.JNSBM_285_16

[30] Cui, C.K., Bian, G.B., Hou, Z.G., Zhao, J., Zhou, H. (2017). A multimodal framework based on integration of cortical and muscular activities for decoding human intentions about lower limb motions. IEEE Transactions on Biomedical Circuits and Systems, 11(4): 889-99. https://doi.org/10.1109/TBCAS.2017.2699189

[31] Sadeghi, S., Maleki, A. (2019). Accurate estimation of information transfer rate based on symbol occurrence probability in brain-computer interfaces. Biomedical Signal Processing and Control, 54: 101607. https://doi.org/10.1016/j.bspc.2019.101607

[32] Wolpaw, J.R., Ramoser, H., McFarland, D.J., Pfurtscheller, G. (1998). EEG-based communication: Improved accuracy by response verification. IEEE Transactions on Rehabilitation Engineering, 6(3): 326333. https://doi.org/10.1109/86.712231

[33] Mackay, D.J.C. (2003). Information Theory, Inference, and Learning Algorithms. Cambridge University Press.

[34] Cover, T.M., Thomas, J.A. (2005). Elements of Information Theory. Wiley.

[35] Cohen, J. (1960). A coefficient of agreement for nominal scales. Educational and Psychological Measurement, 20(1): $37-46$. https://doi.org/10.1177/001316446002000104

[36] Alshbatat, A.1.N., Vial, P.J., Premaratne, P., Tran, L.C. (2014). EEG-based brain-computer interface for automating home appliances. Journal of Computers, 9(9) 2159-2166. https://doi.org/10.4304/jcp.9.9.2159-2166

[37] Alrajhi, W., Alaloola, D., Albarqawi, A. (2017). Smart home: toward daily use of BCI-based systems. in: 2017 Int. Conf. Informatics, Heal. Technol., IEEE, pp. 1-5. https://doi.org/10.1109/ICIHT.2017.7899002

[38] Brennan, C., McCullagh, P., Lightbody, G., Galway, L., Feuser, D., González, J.L., Martin, S. (2015). Accessing tele-services using a hybrid BCI approach. Advances in Computational Intelligence, Springer, Cham, pp. 110123. https://doi.org/10.1007/978-3-319-19258-1 10

\section{APPENDIX}

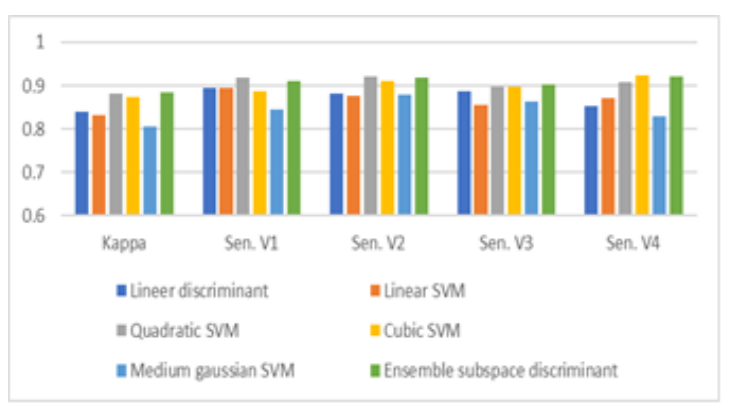

Figure A1. Cohen's kappa coefficient and sensitivity of each vane in 6-sec epochs based on the hold-out strategy

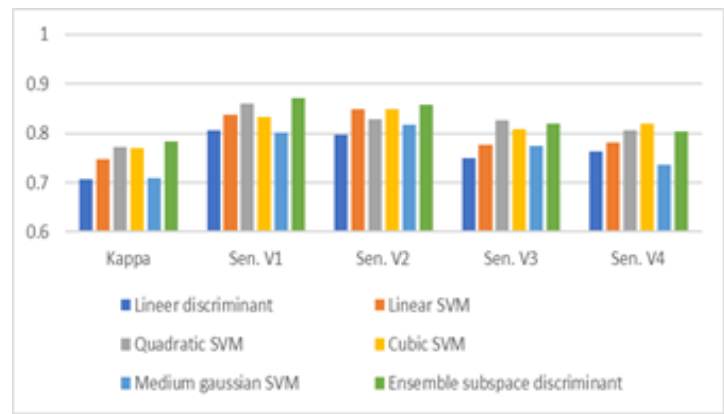

Figure A2. Cohen's kappa coefficient and sensitivity of each vane in 4-sec epochs based on the hold-out strategy

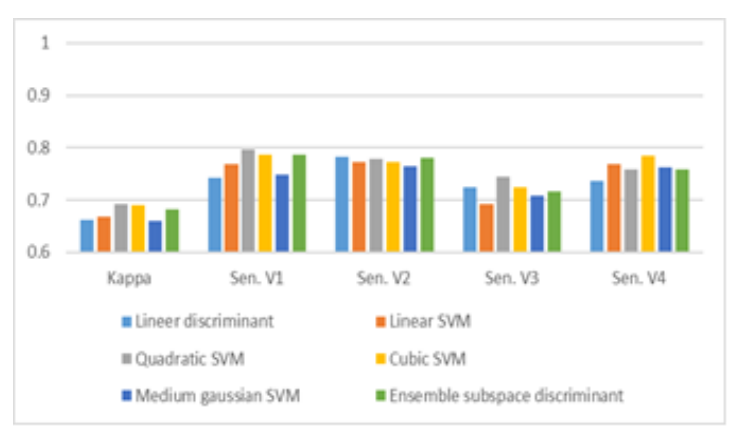

Figure A3. Cohen's kappa coefficient and sensitivity of each vane in 2-sec epochs based on the hold-out strategy 